Canadian University Music Review

Canadian University Music Review

Revue de musique des universités canadiennes

\title{
Cohésion compositionnelle dans Image de Germaine Tailleferre
}

\section{Jacinthe Harbec}

Volume 18, numéro 2, 1998

URI : https://id.erudit.org/iderudit/1014654ar

DOI : https://doi.org/10.7202/1014654ar

Aller au sommaire du numéro

\section{Éditeur(s)}

Canadian University Music Society / Société de musique des universités canadiennes

\section{ISSN}

0710-0353 (imprimé)

2291-2436 (numérique)

Découvrir la revue

\section{Citer cet article}

Harbec, J. (1998). Cohésion compositionnelle dans Image de Germaine Tailleferre. Canadian University Music Review / Revue de musique des universités canadiennes, 18(2), 48-71. https://doi.org/10.7202/1014654ar
Résumé de l'article

L'article présente une étude analytique d'Image pour huit instruments (1918) de Germaine Tailleferre. La démonstration fera la preuve de la cohésion compositionnelle du travail de Tailleferre en se penchant sur deux aspects fondamentaux : le motif et la forme. La démarche analytique proposée consiste à considérer le motif sur divers plans de la composition. La représentation graphique des structures linéaires qui résulte de l'analyse permet d'illustrer comment les notes du motif initial de surface se tiennent à l'arrière-plan de la composition, lesquelles notes s'enchaînent en synchronisme avec les diverses composantes de la structure formelle de l'œuvre.
All Rights Reserved (C Canadian University Music Society / Société de musique des universités canadiennes, 1998
Ce document est protégé par la loi sur le droit d'auteur. L’utilisation des services d'Érudit (y compris la reproduction) est assujettie à sa politique d'utilisation que vous pouvez consulter en ligne.

https://apropos.erudit.org/fr/usagers/politique-dutilisation/ 


\title{
COHÉSION COMPOSITIONNELLE DANS IMAGE DE GERMAINE TAILLEFERRE
}

\author{
Jacinthe Harbec
}

On se souvient de Germaine Tailleferre (1892-1983) en tant que seule femme au sein du Groupe des $\mathrm{Six}^{1}$. Cette compositrice nous a légué à sa mort un héritage considérable de plus de 175 œuvres dont la plupart sont encore inconnues. L'ensemble de sa production couvre une variété de genres et de styles, allant de la musique de scène et de film aux opéras, en passant par les concertos et la musique de chambre et de piano ${ }^{2}$. Son style s'inscrit dans la tradition de la musique française du début du $\mathrm{XX}^{\mathrm{e}}$ siècle, tradition où l'élément motivique joue un rôle prépondérant ${ }^{3}$.

Les caractéristiques du langage compositionnel de Tailleferre apparaissent dès ses premières œuvres. Sa principale source d'inspiration provient de son entourage musical, constitué alors d'un groupe de jeunes musiciens regroupés sous l'appellation de Société des Nouveaux Jeunes", attribuée par leur «bon maître d'Arcueil », Erik Satie. La première participation de Tailleferre dans la série de concerts de ce groupe date du 15 janvier 1918 au Théâtre du VieuxColombier : elle y présentait une suite de deux pièces pour deux pianos, Jeux de plein air (1917) ainsi qu' une Sonatine pour cordes (1917) . En novembre de la même année, Tailleferre terminait une nouvelle composition, Image (1918), destinée à huit instruments : flûte, clarinette, célesta, piano et quatuor à cordes ${ }^{6}$. Cette œuvre, encore plus que les deux précédentes, s'inscrit dans le

1 Le Groupe des Six rassemblait Georges Auric, Louis Durey, Arthur Honegger, Darius Milhaud, Francis Poulenc et Germaine Tailleferre.

2On trouvera plus de détails sur ces compositions dans le catalogue d'œuvres de Tailleferre présenté en annexe 1 dans Jacinthe Harbec, "CEuvres de Germaine Tailleferre : du motif à la forme " (thèse de doctorat, McGill University, 1995), 313-43.

3 Des études récentes confirment que cette caractéristique motivique constitue l'élément intrinsèque de la musique en France au début du $\mathrm{XX}^{\mathrm{e}}$ siècle. Voir par exemple Allen Forte, "La terrasse des audiences du clair de lune : approche motivique et linéaire ", Analyse musicale 16 (juin 1989) : 23-30; Joseph Straus, "Stravinsky's Tonal Axis ", Journal of Music Theory 26, $\mathrm{n}^{\circ} 2$ (1982) : 261-90; finalement, Harbec, « Euvres de Germaine Tailleferre : du motif à la forme ». Des auteurs comme Donald R. McLean et Brian Alegant travaillent présentement à développer une théorie autour de cette question dont les principes dominants ont été énoncés dans la communication " On the Nature of Enlargement 》 (conférence annuelle de la Society for Music Theory, New York, 4 novembre 1995); à paraître dans la revue de la société.

4La Société des Nouveaux Jeunes était alors formée de Durey, d'Auric, d'Honegger, de RolandManuel et de Tailleferre. À l'exception de Roland-Manuel, ces musiciens feront partie du futur Groupe des Six.

5Les deux mouvements de la Sonatine pour cordes constitueront les deux premiers des trois mouvements de l'œuvre Quatuor terminée en 1919.

6 Il existe une transcription pour piano à quatre mains réalisée par la compositrice qui porte le titre 
courant esthétique défini par ce groupe de jeunes compositeurs. Aussi retrouvons-nous à la fois la clarté dans l'écriture musicale, héritée des maîtres clavecinistes français, et l'exotisme omniprésent dans les théâtres et musichalls de l'époque. Bien que l'œuvre Image ait été composée au début de la carrière de Tailleferre, ce clin d'œil musical de six minutes affiche déjà les éléments stylistiques qui caractériseront ses compositions à venir.

L'analyse suivante d'Image tente de démontrer la cohésion compositionnelle présente dans la musique de Tailleferre en se basant sur un de ses éléments intrinsèques, à savoir le motif. Cette démarche nous a été inspirée par quelques propos tenus par Tailleferre elle-même dans ses entrevues. Dans un de ces entretiens, elle définit le processus de composition comme étant « un travail de pensée, une idée fixe ${ }^{7}$. Cette 《idée fixe » dont elle parle peut aussi bien correspondre à l'idée globale que l'on se fait d'une œuvre qu'à une idée musicale de base.

Cet autre propos de Tailleferre nous informe davantage sur ce qu'elle entend par « travail de pensée » en composition :

Premièrement, j'utilise ma tête - quels sons, quelle forme donner, comment la faire. Puis, je cherche un thème au piano. Si j'en trouve un, je commence à travailler, à l'écrire, et à partir de ce moment, j'utilise beaucoup le piano. Mais non au début. Si le thème vient, les choses viennent habituellement très vite ${ }^{8}$.

Ce témoignage démontre bien le lien étroit entre la vision d'ensemble de l'œuvre - son organisation harmonique et formelle à grande échelle - et le matériau de départ que nous appelons « idée fondamentale ». Nous pourrions ainsi considérer que l'idée fondamentale, une fois « fixée », reste obstinément en mémoire tout au long de l'accomplissement de l'idée globale, soit de la composition de l'œuvre entière. Au moyen d'une technique de « transformation motivique continue », les motifs propres à l'idée initiale et fondamentale sont à la base de l'écriture du thème et de toutes les formulations thématiques subséquentes nécessaires à la construction complète de l'œuvre. Ainsi, le parcours qu'emprunteront les multiples transformations de l'idée fondamen-

d'Image pour huit instruments, alors que le titre de la partition pour petit ensemble se limite à Image. Ces deux titres, correspondant aux versions respectives, portent souvent à confusion. Les deux versions ont été publiées chez Chester : en 1920 dans le cas de la version pour petit ensemble, en 1921 dans le cas de la version pour piano à quatre mains. Nous serions portée à croire que l'effectif instrumental particulier de cette œuvre a constitué une contrainte pour pouvoir l'inclure dans l'un des programmes de la série de concerts de la Société des Nouveaux Jeunes. En effet, on ne sait pas si Image a été créée à cette époque. Il y a cependant eu une première américaine, donnée le 7 mai 1992 par l'ensemble de la University of California at Santa Cruz sous la direction de Nicole A. Paiement.

7Citée dans Hélène Jourdan-Morhange, Mes amis musiciens (Paris : Librairie Gallimard, 1943), 159.

8 « First I use my head - what sounds, what form to give, how to do it. Next I look for a theme at the piano. If I find one, I begin to work, to write it, and from then on I use the piano a lot. But not at the beginning. If the theme comes, things usually happen very quickly. » Entretien avec Laura Mitgang, "One of "Les Six" Is Still At Work », New York Times, 23 mai 1982 (traduction par l'auteure). 
tale déterminera la forme de l'œuvre : cette dernière représente l'étape finale de l'accomplissement de l'idée fondamentale'.

C'est ce modèle d'analyse, basé sur les liens motiviques et thématiques, que nous proposons dans l'étude sur la cohésion compositionnelle d'Image. Avant d'amorcer l'analyse proprement dite, nous discuterons de la valeur sémantique potentiellement attribuable au titre Image et de la façon dont elle s'applique tant sur le plan de la vision globale de la composition que sur celui de sa structure formelle. Puis, nous définirons l'idée fondamentale de l'œuvre et les motifs caractéristiques capables de générer l'ensemble de la composition. Dans cette partie, nous expliquerons la démarche analytique utilisée, basée sur le motif. Ensuite, nous appliquerons les principes de l'analyse motivique à Image, analyse qui sera effectuée tout d'abord à un niveau de surface. Cette analyse progressera en synchronisme avec la démonstration de la structure formelle de l'œuvre. La cohésion qui ressortira de cette discussion sera davantage mise en valeur lorsque nous aborderons, en dernier lieu, le sujet du motif au niveau profond de la composition ${ }^{10}$. Cette démarche révélera de manière significative comment le matériel musical de départ, l'idée fondamentale, agit comme force unificatrice au sein des différentes sections de l'œuvre.

\section{Valeur sémantique, vision globale et forme}

Une lecture approfondie de l'œuvre de Tailleferre laisse entrevoir que le titre Image ne relève pas nécessairement de l'imaginaire, mais plutôt de la sémantique du terme, à savoir l' " image » formée par l'objet immobile. Image devrait en fait être perçue comme l'équivalent musical d'un cliché instantané constituée de divers plans en relief. En effet, la surface sonore de Image est sectionnée par une succession d'unités musicales clairement délimitées que nous appelons «plans sonores ». Cet aspect introduit dans la composition un effet de " construction fragmentaire », provoquant ainsi une brisure dans le flux musical puisque les unités s'ajoutent les unes aux autres dans un enchaînement brusque sans lien transitoire ${ }^{11}$. Ajoutons que la composition est fondée

\footnotetext{
9Dans notre démarche analytique, nous nous sommes inspirée, entre autres, d'un des concepts énoncés par Arnold Schönberg, notamment celui de Grundgestalt. La Grundgestalt implique la notion d' « idée fondamentale » ou de « motif fondamental » qui, par le biais du « développement par variation ", génère toutes les formulations thématiques et, par extension, la composition entière. Pour une présentation de cette notion, voir, entre autres, Arnold Schönberg, Le style et l'idée, écrits réunis par Leonard Stein, traduit de l'anglais par Christiane de Lisle (Paris : Buchet/Chastel, 1977), 304. Pour plus de détails sur les différentes interprétations de ce concept, voir Charlotte M. Cross, «Three Levels of "Idea" in Schoenberg's Thought and Writings ", Current Musicology 30 (1980) : 24-36; et David Epstein, Beyond Orpheus, (Cambridge, Mass. : MIT Press, 1979), chapitre 2 et appendice A.

10Rudolph Reti et Hans Keller, des successeurs de Schönberg, ont aussi senti le besoin de développer le concept du « développement par variation » dans un niveau de structure plus profond que celui de surface. Par contre, on note dans l'ouvrage théorique de Reti, Thematic Process in Music (New York : Macmillan, 1951), que la méthode traite surtout le matériel de niveau purement de surface sans compter qu'elle s'intéresse principalement aux structures mélodiques, délaissant ainsi les structures harmoniques. Cette dernière remarque s'applique aussi bien à la méthode de Keller qu'à celle de Reti. Pour une application plus récente de la théorie du «développement par variation », voir Walter Frisch, Brahms and the Principle of Developing Variation, California Studies in 19th-Century Music, vol. 2 (Berkeley : University of California Press, 1984).

11 Un phénomène semblable se retrouve dans le ballet Parade de Satie, composé en 1917, soit un
} 
en grande partie sur des motifs d'ostinatos qui entraînent la dissolution de toute syntaxe harmonique comme nous l'entendons traditionnellement. Ces éléments, mis ensemble, contribuent à créer l'effet d'immobilité qui émane d'Image.

Chacun des plans sonores d'Image est tapissé de diverses textures ou strates sonores composées de mélodies, d'ostinatos et de notes pédales qui donnent lieu à une structure polytonale. Dans le graphique A de l'analyse linéaire d'Image qui se trouve en appendice ${ }^{12}$, chaque strate sonore est représentée sur une portée différente :

1. dans la voix de basse se placent les notes pédales et les ostinatos;

2. les ostinatos de registre supérieur et les notes d'accompagnement figurent dans la voix intermédiaire, au-dessus de la basse;

3. les lignes plus mélodiques sont indiquées dans les deux autres voix, dont la supérieure est réservée à la contre-mélodie, que nous appelons « cantus » (nous verrons plus loin ce que nous entendons précisément par « cantus »); quant à la deuxième voix à partir du haut, elle représente la « mélodie » proprement dite.

L'union des diverses voix mélodiques dans une unité donnée se manifeste en plan sonore. Comme nous l'avons mentionné, le flux musical d'Image est constamment sectionné par un changement brusque de l'ostinato, ce qui amène un nouveau plan sonore. Au fur et à mesure que l'on avance dans le temps, les 15 plans sonores distincts (voir le schéma formel placé au-dessus des graphiques) s'ajoutent les uns aux autres jusqu'à la formation complète de l'Image, à l'instar du processus de création d'une toile picturale.

Ce façonnement de la composition d'Image se manifeste en deux stades. L'arrivée du point d'orgue aux deux tiers de la partition, telle qu'elle est

an avant Image. L'aspect de construction fragmentaire de Parade a fait l'objet d'une grande part de la thèse de maîtrise de l'auteure, "Parade, les influences cubistes sur la partition musicale d'Erik Satie " (McGill University, 1987).

12Comme il n'est impossible de reproduire la partition entière, nous jugeons indispensable de représenter sous forme de graphique une lecture de niveau intermédiaire de l'œuvre qui en illustre les structures linéaires les plus représentatives. Le graphique $A$ de niveau intermédiaire, qui ne sera pas expliqué en détail, sert ici d'appui à l'interprétation du graphique B, de rang plus profond, auquel nous nous référerons principalement dans cette démonstration. Compte tenu du langage posttonal de la musique de Tailleferre, les représentations graphiques ne suivent pas les règles de l'analyse schenkérienne, cette dernière étant conçue pour l'étude d'œuvres tonales. Il serait plus juste d'identifier cette démarche en tant qu'analyse des structures linéaires où, malgré l'absence de la syntaxe tonale, il est possible de représenter la conduite des voix résultant du contrepoint de cette composition de type diatonique. En ce qui a trait aux critères de regroupement aux fins de représentation graphique, ils respectent en général ceux énoncés par Allen Forte dans « New Approaches to the Linear Analysis of Music ", Journal of American Musicological Society 41, $\mathrm{n}^{0} 2$ (été 1988) : 346, et "La terrasse des audiences du clair de lune : approche motivique et linéaire », 24. En résumé, le regroupement s'effectue de pair avec les motifs et les phrases mélodiques définis par plusieurs paramètres dont le rythme, le contour et le registre, tout en prenant en considération les lignes d'accompagnement et le mouvement des notes de basse qui les soutiennent. Pour d'autres points de vue sur le sujet ou pour consulter une application semblable, voir entre autres Joseph Straus, « Stravinsky's Tonal Axis » et V. Kofi Agawu, «Schenkerian Notation in Theory and Practice », Music Analysis 3, $\mathrm{n}^{\circ} 3$ (octobre 1989) : 275-301. 
illustrée dans le schéma formel, est responsable de cette division. À chacun des deux stades, Tailleferre semble prêter un sens distinct au mot « image » :

1. le premier stade renvoie au processus de création d'un objet, c'est-à-dire à la formation graduelle de l' « image » jusqu'à son achèvement;

2. le deuxième stade fait référence à la réflexion de l' " image », c'est-àdire à sa reproduction inversée.

Aussi, dans un laps de temps deux fois plus court (voir le nombre de temps indiqué dans le schéma formel), le deuxième stade de l'œuvre va procéder à la déconstruction jusqu'à l'effacement presque complet du matériau sonore.

Dans un langage plus théorique et moins imagé, nous dirions que l'œuvre présente une structure en forme d'arche, ou ce que nous appelons la grande forme rondo. Le schéma formel reproduit en appendice montre les particularités de cette forme. Elle se divise en cinq sections principales qui reproduisent, d'après le matériel mélodique-motivique, le schéma suivant : $A-B-C-B^{\prime}-A^{\prime}$. Chacune de ces sections est redivisée en 15 plans sonores (identifiés par « pl. s. » dans le schéma) rassemblant plusieurs unités qui se regroupent à leur tour sous une forme distincte.

\section{Définition de l'idée fondamentale et des motifs caractéristiques}

Le lien entre le début et la fin d'Image indique que Tailleferre présente dès ses premières mesures des indices quant à la définition de l'idée fondamentale de l'œuvre. L'exemple 1 nous informe des caractéristiques des motifs reliés à l'idée fondamentale déjà camouflée dans la première unité de huit mesures.

Cette unité identifiée « $\mathrm{a}^{1}$ », reproduite à l'exemple 1 , se compose de la répétition d'une courte mélodie de quatre mesures qui se déploie aisément à partir d'un fond sonore stable constitué de notes pédales. Cette première ligne mélodique rassemble quatre notes seulement (do, ré, fa et sol, suggérant le pentatonique) et met en valeur deux motifs caractéristiques :

1. le motif « $\mathrm{R} »$, constitué de la répétition de la note ré (exemple 1a, mes. 1);

2. le motif « $\mathrm{B}$ », constitué du geste de broderie ré-do-ré (mes. 1-2).

Après les motifs « $\mathrm{R}$ » et « $\mathrm{B}$ », la ligne effectue une montée jusqu'à sol, pour ensuite revenir à son point de départ en refaisant dans le sens inverse le tracé de la ligne initiale, telle une «image " réfléchie dans un miroir. Le tracé en forme d'arche résultant de cette mélodie se dessine à partir de notes piliers dont l'ensemble correspond à une structure mélodique fondamentale. Nommé «I.F. », pour idée fondamentale, ce motif est illustré à l'exemple le.

Dans l'accord d'accompagnement de cette mélodie (exemple 1a), Tailleferre fait allusion à la pratique tonale en superposant l'harmonie de sol septième de dominante à une pédale pseudo-tonique formée de la quinte juste $d o-$ sol $^{13}$. En fait, cette structure harmonique rassemble les quatre notes de la

13Dans son analyse harmonique d'Image ( "The Harmonic Idiom in the Works of "Les Six" » [thèse de doctorat, Boston University Graduate School, 1963], 445-46), Richard B. Bobbitt fait état de ce lien de dominante/tonique dans une même structure harmonique en précisant qu'il ne relève pas d'une pratique nouvelle, étant notamment présent chez Mozart. Cette comparaison ne nous paraît pas 


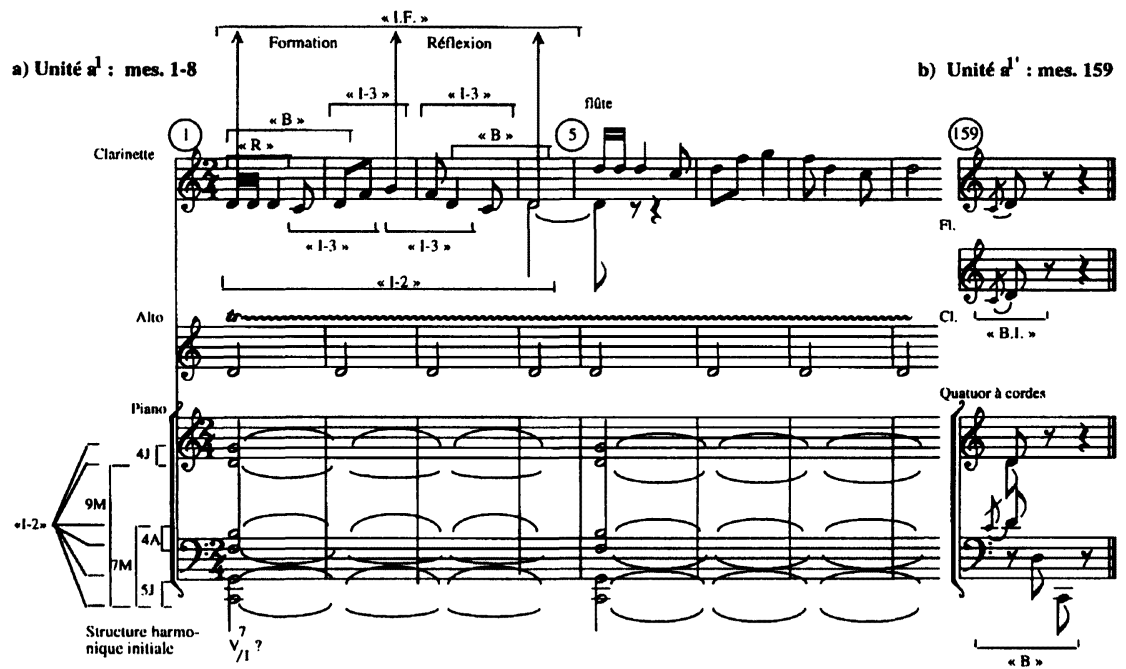

c) Motifs mélodiques
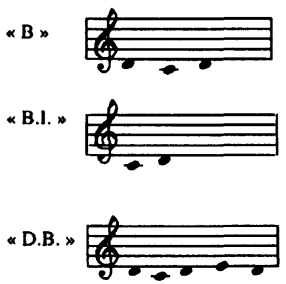

« D.B.I.

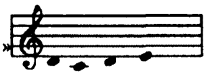

“ $R$ *

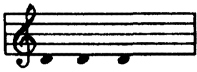

d) Ensembles de notes
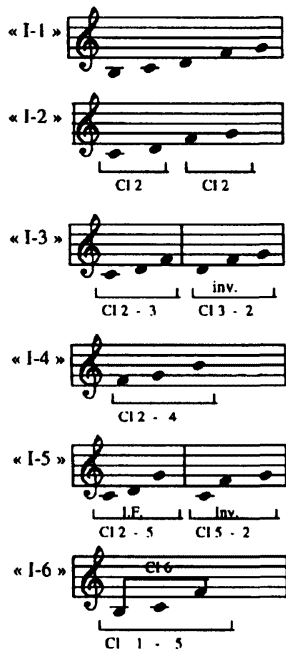

e) Motif de l'idée fondamentale
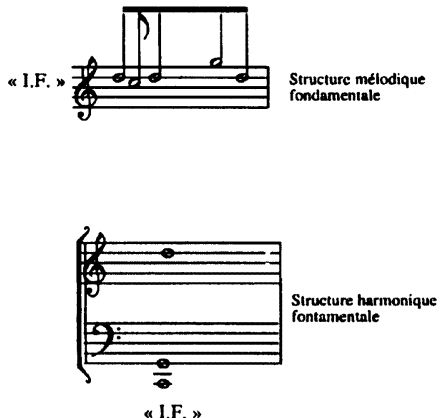

Exemple 1 : Motifs caractéristiques d'Image

mélodie plus une (si). Cette note supplémentaire n'est pas de moindre importance, car elle ajoute à l'intervalle de neuvième majeure d'autres dissonances provoquées par les intervalles de septième majeure do-si et de quarte augmen-

appropriée parce que les deux exemples de Mozart sont le résultat d'un retard. Plus précisément, malgré la présence des notes de l'accord de dominante exerçant le retard sur l'harmonie de tonique, c'est tout de même l'harmonie de tonique qui est perçue par notre oreille. Par conséquent, la fonction harmonique au moment du retard est uniquement de tonique et non simultanément des deux fonctions de tonique/dominante. Dans Image de Tailleferre, le contexte dans lequel s'insère cette superposition interdit toute interprétation de retard, ce qui mène à considérer cette structure harmonique comme étant réellement formée d'une superposition de deux accords issus de la même collection de notes de do majeur. 
tée $f a-s i$. Nous verrons, en progressant dans l'analyse de l'œuvre, comment ces intervalles contribuent à sa cohésion compositionnelle et à son organisation formelle.

Dans l'organisation mélodique et rythmique de cette première unité thématique de huit mesures (exemple 1a), nous percevons une insistance sur le son ré. En plus d'être la note initiale et finale de la mélodie et aussi de faire partie de l'accord accompagnateur, le ré est à la source d'un trille que l'alto exécute tout au long de l'unité (exemple 1a, mes. 1-8). Cette importance accrue, qui lui est indéniablement accordée dès les premières mesures et qui, comme nous le verrons, sera soutenue pendant le déroulement de l'œuvre, nous porte à attribuer au son ré le rôle de note mélodique structurelle. Celle-ci repose sur une double note de basse structurelle composée d'une quinte harmonique do-sol, et l'ensemble de notes do-sol-ré ainsi créé forme la structure harmonique fondamentale apparaissant à l'exemple 1e. Il faut noter que les notes extrêmes ré-do de cet accord par étagement de quintes de l'exemple 1e reproduisent harmoniquement les notes qui réalisent le motif de broderie de la mélodie retranscrite à l'exemple $1 \mathrm{a}$.

\section{Présentation des motifs caractéristiques}

L'analyse des huit premières mesures d'introduction d'Image nous permet de définir les motifs caractéristiques qui constituent les éléments de base de l'œuvre. La liste de ces motifs figure aux exemples $1 \mathrm{c}$ et $1 \mathrm{~d}$. Les motifs apparaissant à l'exemple $1 \mathrm{c}$ découlent tous du motif de broderie (« B »). Ils constituent en quelque sorte différentes façons de réaliser la broderie comme, par exemple, la broderie incomplète (« B.I. »), la double broderie (« D.B. ») et la double broderie incomplète («D.B.I. »). Même le motif « $R$ » peut être perçu, par extension, comme une variante de la broderie si nous considérons que sa fonction consiste à prolonger un son. Ces motifs subissent, au cours de la composition, diverses transformations : la transposition, l'inversion (en miroir), la rétrogradation (à rebours), la rotation, ou encore les quatre simultanément ${ }^{14}$.

Les motifs de l'exemple $1 \mathrm{~d}$ présentent des ensembles de notes classés par leur contenu spécifique d'intervalles. Ils méritent une entité sonore propre sans que la notion de contour ne soit une condition sine qua non. La définition de ces motifs a été inspirée de la théorie des ensembles ${ }^{15}$, dont nous allons présenter quelques notions préliminaires.

14 Pour éviter d'alourdir l'analyse, nous ne spécifierons pas chacune des transformations effectuées sur les motifs discutés.

15Milton Babbitt est celui qui a présenté le premier les principes de la théorie des ensembles des hauteurs de son (en anglais set theory) dans les articles « Twelve-Tone Invariants as Compositional Determinants », The Musical Quarterly 46, $\mathrm{n}^{\circ} 2$ (avril 1960) : 246-59, et « Set Structure as a Compositional Determinant », Journal of Music Theory 5, $\mathrm{n}^{\circ} 2$ (1961) : 72-94. Forte, dans The Structure of Atonal Music (New Haven, Conn. : Yale University Press, 1973), a su réunir les divers concepts de cette théorie des ensembles qui ont suivi les articles de Babbitt. Pour un résumé explicatif de la théorie des ensembles ainsi qu'un historique de son développement, voir le chapitre d'introduction dans Janet Schmalfeldt, Berg's Wozzeck : Harmonic Language and Dramatic Design (New Haven, Conn. : Yale University Press, 1983), 1-28. Pour l'application de la théorie des ensembles tout en considérant le 
D'abord, l'approche théorique utilisée ici repose sur une configuration numérique. Chaque note est identifiée par un nombre entier à l'intérieur du modulo 12 (de 0 à 11) qui en représente la classe de hauteur ${ }^{16}$. Aussi, sans nécessairement considérer la notion du registre, chaque hauteur de son de l'échelle chromatique correspond à $0,1,2, \ldots, 11$, où 0 équivaut à la note $d o$. Ainsi, toute note de même nom est porteuse d'un nombre propre, indépendamment du registre dans lequel elle figure. Par exemple, deux notes $d o$ situées à une octave de distance ont la même classe $0^{17}$.

Dans le même ordre d'idées, la distance entre deux classes de hauteurs est identifiée par une classe numérique d'intervalle à l'intérieur du modulo 12 . Dans ce cas, chaque classe intervallique (indiquée désormais par $\mathrm{CI}$ ) correspond au nombre de demi-tons compris entre deux classes de hauteurs. Ainsi, toute distance équivalant à la seconde mineure $(2 \mathrm{~m})^{18}$ correspond à la CI 1 , la seconde majeure $(2 \mathrm{M})$ à la CI 2 , la tierce mineure $(3 \mathrm{~m})$ à la CI 3 , etc. Par ailleurs, et c'est ce que nous retiendrons surtout, les CI peuvent se limiter au modulo $6,{ }^{19}$ acceptant comme équivalent leur renversement et leur redoublement respectif. La liste suivante offre le contenu de chaque classe intervallique, incluant le redoublement de chaque intervalle :

CI 0 comprend la $1 \mathrm{~J}$ et la $8 \mathrm{~J}$;

CI 1 comprend la $2 \mathrm{~m}$ et la $7 \mathrm{M}$;

CI 2 comprend la $2 \mathrm{M}$ et la $7 \mathrm{~m}$;

CI 3 comprend la $3 \mathrm{~m}$ et la $6 \mathrm{M}$;

CI 4 comprend la $3 \mathrm{M}$ et la $6 \mathrm{~m}$;

CI 5 comprend la $4 \mathrm{~J}$ et la $5 \mathrm{~J}$;

CI 6 comprend la $4 \mathrm{~A}$ et la $5 \mathrm{D}^{20}$.

contexte posttonal mais diatonique des œuvres étudiées, voir Richard S. Parks, The Music of Claude Debussy, Composers of the Twentieth Century (New Haven, Conn. : Yale University Press, 1989); et James M. Baker, The Music of Alexander Scriabin, Composers of the Twentieth Century (New Haven, Conn. : Yale University Press, 1986).

16De l'anglais pitch class, traduit en français par Forte lui-même dans «La terrasse des audiences du clair de lune : approche motivique et linéaire ». Mentionnons la précision qu'apporte Célestin Deliège sur cette notion : « il s'agit d'un étiquetage de classes de hauteurs et non de hauteurs réelles »; voir « La Set-Theory ou les enjeux du pléonasme», Analyse musicale 17 (octobre 1989) : 66.

17 Ce qui correspond à l'octave equivalence; voir Forte, The Structure of Atonal Music, 2-3. Pour des définitions détaillées, consulter le premier chapitre de Joseph N. Straus, Introduction to Post-Tonal Theory (Englewood Cliffs, N.J. : Prentice Hall, 1990), 1-15.

$18 \mathrm{Au}$ cours de l'analyse, lorsqu'il sera indispensable de référer à l'intervalle précis, nous utiliserons ce symbole indiqué entre parenthèses : « $m$ » pour mineur, « $M$ » pour majeur, « $D$ » pour diminué, « $A$ » pour augmenté et « $J$ » pour juste.

$19 \AA$ À ce sujet, voir Forte, The Structure of Atonal Music, 14.

20 Il faut lire ici toute distance entre deux notes qui correspond en tant que résultat sonore à l'un des intervalles mentionnés et à son redoublement. Pour plus de détails, voir les sections « Unordered Pitch-Class Interval » et «Interval Class » dans Straus, Introduction to Post-Tonal Theory, 7-8. Forte se sert des classes intervalliques pour identifier la teneur en classes d'intervalles d'un ensemble distinct qu'il nomme "vecteur intervallique ". La classification des ensembles qui est établie selon la notice vectorielle est représentée dans la table de l'appendice 1 de The Structure of Atonal Music, 179-81. Dans la présente analyse, nous n'utilisons pas la nomenclature de Forte, nous limitant ici à quelques ensembles les plus caractéristiques. 
La référence à la théorie des ensembles dans l'étude d'Image se restreint à la classification et à l'identification de certaines structures motiviques caractéristiques regroupées à l'exemple $1 \mathrm{~d}$. Le premier ensemble de cet exemple reprend toutes les notes utilisées dans la première unité de l'introduction. Conformément à la théorie des ensembles, ces notes sont retranscrites de façon ascendante, de manière à ce que se suivent les plus petits intervalles à l'intérieur de l'octave, et de manière à ce qu'il se déploie la plus petite distance entre les notes extrêmes de l'ensemble ${ }^{21}$. Nous nommons «I-1 " l'ensemble de notes de référence : le «I $\mathrm{I}$ réfère à la première lettre du titre Image, et le « 1 » indique qu'il est l'ensemble de notes de départ, son ensemble initial.

L'ensemble « I-1 » génère d'autres ensembles plus petits dont les plus caractéristiques, c'est-à-dire ceux sur lesquels sera basée la discussion suivante, succèdent à l'ensemble «I-1 » et sont numérotés, à partir de la lettre " I », de 2 à 6 . Dans cette succession, nous avons tenté d'associer une des classes d'intervalles caractéristiques de l'ensemble au rang que ce dernier occupe dans la liste. Aussi, les ensembles «I-2 » à « I-5 », qui commencent tous par la CI 2, présentent comme dernier intervalle les classes allant de 2 à 5 respectivement. Par exemple, l'ensemble «I-2 » se termine par la CI 2, l'ensemble «I-3 » par la CI 3, et ainsi de suite jusqu'à l'ensemble « I-5 ». Pour ce qui est de l'ensemble «I-6 », il débute par la CI 1 et la somme de la succession des deux CI est égale à la CI 6.

Dans les cas où deux ensembles se tiennent sur la même portée, comme « I-3 » par exemple, ces ensembles contiennent les mêmes classes d'intervalles, mais ces dernières sont inversées l'une par rapport à l'autre ${ }^{22}$. Pour ce qui est de l'ensemble «I-5 », il reproduit l'ensemble «I.F. » présenté à l'exemple 1e, tous les deux possédant la même spécificité intervallique. L'appellation «I.F. » est alors attribuée à l'ensemble «I-5 » seulement si cet ensemble est formé exclusivement des notes do, ré et sol.

Notre démonstration de la cohésion d'Image est basée sur ces motifs ou ensembles ${ }^{23}$ présentés à l'exemple 1 . Elle se fera à partir d'extraits représentatifs de chaque section formelle du premier stade de la composition, à savoir des sections A, B et C. L'étude motivique et formelle du premier stade nous préparera à la discussion de la grande structure sous-jacente de l'œuvre. Nous verrons comment, à l'échelle de l'œuvre, Image s'est développée et formée à partir de son matériel initial qui comprend notamment :

1. les motifs de la première mélodie (exemples $1 \mathrm{a}$ et $1 \mathrm{c}$ );

2. le motif de l'idée fondamentale « I.F. » (exemple 1e);

3. les six motifs faits des ensembles de notes « $I »($ exemple $1 \mathrm{~d})$.

$21 \mathrm{Ce}$ qui correspond à la notion du normal order. Pour des explications supplémentaires, voir Straus, Introduction to Post-Tonal Theory, 27-30.

22L'inversion intègre le sens adopté dans la théorie des ensembles. Lorsqu'un ensemble de trois hauteurs de son est inversé, la succession des intervalles se trouve simplement interchangée.

23 Les termes « motif » ou « ensemble » sont utilisés indifféremment. 


\section{Démonstration de la cohésion compositionnelle}

\section{Section A}

Le matériel d'introduction de l'œuvre est présenté dans la section A qui se divise en deux unités : « $\mathrm{a}^{1}$ » et « $\mathrm{a}^{2}$ » (voir en appendice le schéma formel dans la partie supérieure des représentations graphiques). Le changement d'unité coïncide habituellement avec l'intervention d'une nouvelle strate sonore qui procure un certain effet de brisure propre à la construction fragmentaire dont nous avons parlé précédemment. Aussi, le caractère plutôt mélodique des huit mesures de l'unité « $\mathrm{a}^{1} »$, émis par la strate supérieure (exemple 1a : clarinette, puis flûte), est interrompu par l'arrivée soudaine d'une strate faite de structures harmoniques jouées au célesta (exemple $2 b$ ). Ce changement brusque, effectué sur la même strate d'accompagnement du piano, signale le début d'une deuxième unité appelée « $\mathrm{a}^{2} »$ (mes. 9-12), à l'intérieur du plan sonore 1 (mes. 1-12).

a) Unité $\mathbf{a}^{1}$

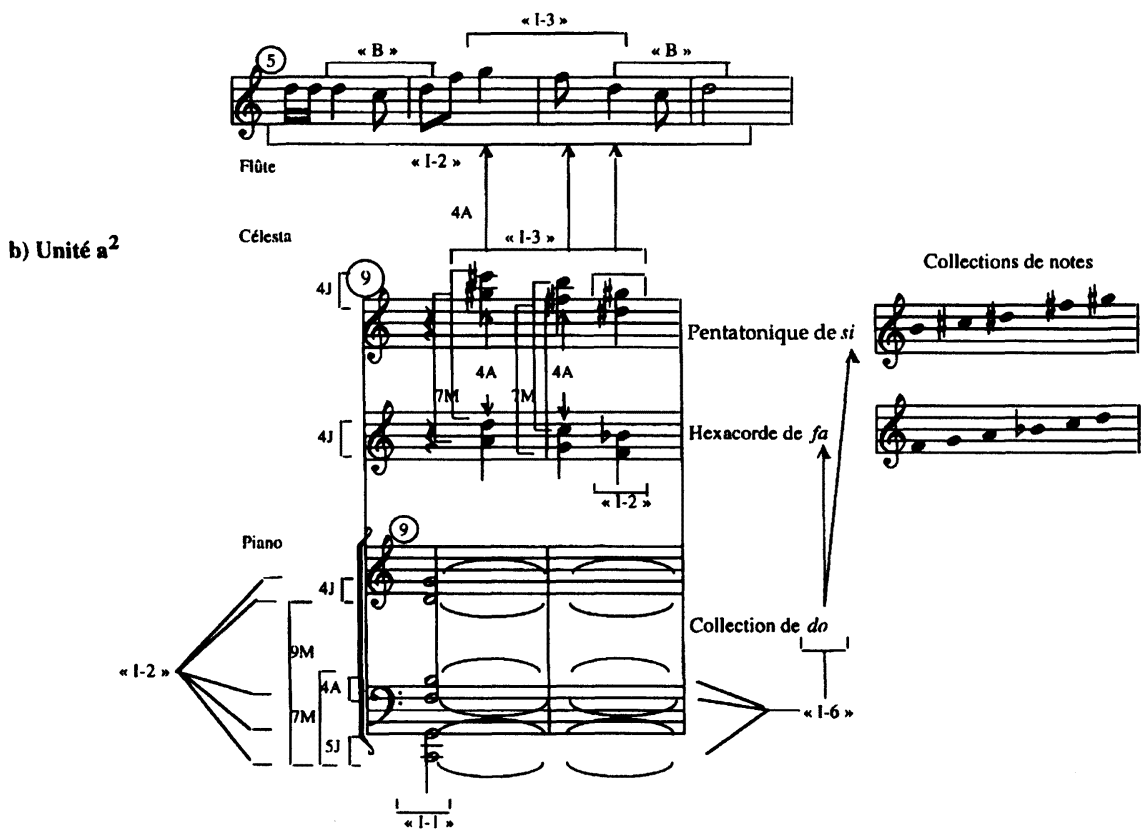

Exemple 2 : Motifs caractéristiques de la section A

La cohésion entre les unités « $\mathrm{a}^{1}$ » et « $\mathrm{a}^{2}$ » apparaît lorsqu' on attribue à chacune des notes d'un ensemble donné le rôle de centre de référence d'une collection de notes distincte ${ }^{24}$. Dès la deuxième unité de l'introduction, l'unité « $\mathrm{a}^{2}$ » illustrée à l'exemple 2, Tailleferre donne à chacune des notes de l'en-

24La collection de notes représentée par le centre référentiel peut aussi bien faire partie d'un mode tonal majeur ou mineur ou d'un des modes ecclésiastiques. 
semble « I-6 » (à droite de l'exemple musical) ce pouvoir de représenter une collection entière. Plus précisément :

1. l'ensemble des notes qui compose l'enchaînement des quartes justes harmoniques de la portée supérieure du célesta provient de la pentatonique de $s i$, telle qu'elle est définie dans la partie de droite de l'exemple $2 b$;

2. l'ensemble de notes qui sert à l'autre enchaînement de quartes justes de la portée inférieure du célesta forme l'hexacorde diatonique de $f a$.

En considérant l'accord accompagnateur de la partie de piano, qui est la reprise de l'accord initial, cette unité « $\mathrm{a}^{2}$ » superpose trois collections dont les centres sont $d o, f a$ et si. Leur union reproduit l'ensemble «I-6 » dont les notes sont puisées à même la structure harmonique initiale « I-1 ».

Outre cette relation entre les collections de notes, mentionnons que la voix supérieure de la progression d'accords du célesta reproduit le tracé rétrograde du premier motif mélodique, l'ensemble «I-3», tel qu'il a été entendu à la fin de la première unité (exemple 2a). La reprise immédiate de ce motif dans la voix supérieure du célesta procure un effet d'écho entendu à la quarte augmentée supérieure. L'exemple $2 \mathrm{~b}$ indique cette transposition de quarte augmentée et montre que cet intervalle, avec la septième majeure, est caractéristique des structures harmoniques qui soutiennent le motif mélodique « I-3 » de la voix supérieure.

En plus de la cohésion motivique, la section A révèle une autre caractéristique de l'œuvre reliée à la notion de temps. Plus précisément, l'enchaînement de la première à la deuxième unité de la section A se réalise à l' " image » de l'œuvre, à savoir en deux stades (dont l'un est plus mélodique que l'autre) et dans la même proportion de longueur de deux pour un.

\section{Section $B$}

Après les 12 mesures de la section $\mathrm{A}$, le piano amorce un motif d'ostinato représentatif de la section B de la composition (exemple 3c). À ce moment précis, une impression d'un véritable début apparaît, comme si, au premier abord, le matériel de la section A pouvait être enlevé sans nuire à l'organisation formelle de l'œuvre. Mais la section A demeure toutefois indispensable à la cohérence motivique que forme Image, tenant lieu d'introduction aux motifs caractéristiques de l'œuvre.

La section $\mathrm{B}$, ou le plan sonore 2, commence par quatre mesures d'introduction (mes. 13-16) reposant exclusivement sur les notes de l'accord du piano (exemple 3c). Toujours présenté au piano, l'accord se compose premièrement de notes pédales réparties dans les voix extrêmes : la voix de basse conserve la même quinte do-sol soutenue pendant la section A ou le plan 1; la voix supérieure emprunte l'appoggiature do-ré qui venait d'être exécutée à la flûte. Dans la voix intermédiaire se déploie un ostinato ressemblant à un arpège brisé qui garde en commun avec l'accord précédent les notes sol-ré, auxquelles la note la est ajoutée. La partie de piano crée ainsi une strate d'accompagnement qui rassemble les notes de l'ensemble «I-2 », do-sol-ré-la. En comparaison avec l'ensemble «I-2» fa-do-sol-ré du plan 1, celui du plan 2 est une transposition à la quinte juste supérieure, comme l'indique l'exemple $3 \mathrm{~d}$. 
a) Unité a ': mélodie d'introduction

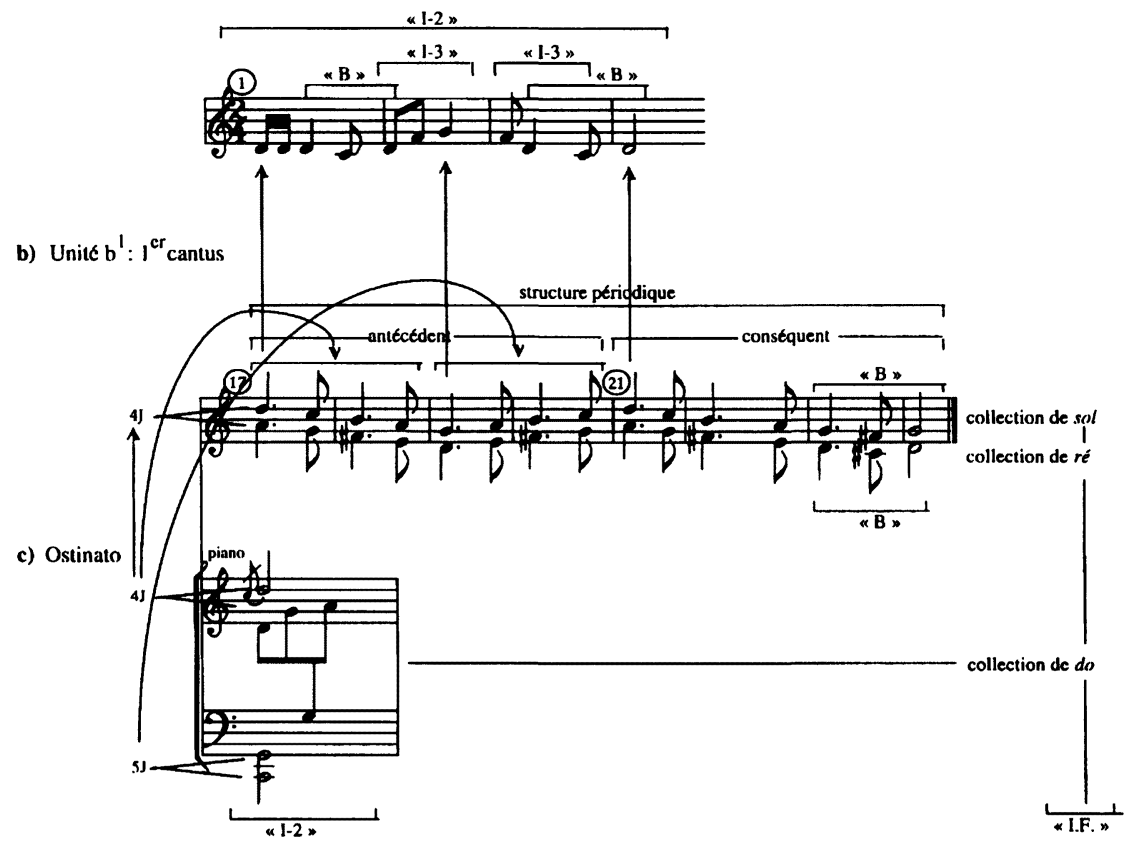

d) Ensembles de notes

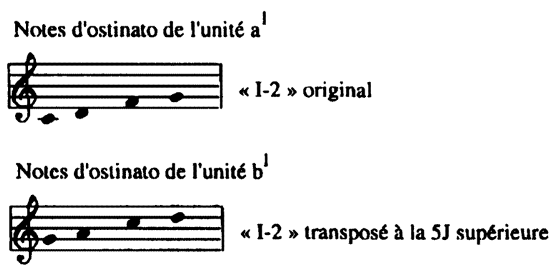

Exemple 3 : Motifs caractéristiques de la section B, $1^{\text {re }}$ unité

Une fois le motif d'ostinato établi, la partie mélodique du plan sonore 2 fait son entrée à la mesure 17 (exemple 3b). Cette ligne mélodique n'agit pas vraiment comme un thème dans le sens classique du terme ${ }^{25}$. Dans le présent contexte, nous désignons par « cantus » la mélodie à la mesure 17 de la section

25Le mot «thème » adopte ici le sens attribué par l'école formelle d'Arnold Schönberg, à savoir, le résultat d'une élaboration du matériel mélodique-motivique en synchronisme avec les enchaînements harmoniques qui se réalisent selon les normes syntaxiques tonales. Pour des explications supplémentaires, voir Schönberg, Fondements de la composition musicale, édition établie par Gerald Strang avec la collaboration de Leonard Stein, traduit de l'américain par Dennis Collins (Paris : Jean-Claude Lattès, 1987); Erwin Ratz, Einführung in die musikalische Formenlehre, $3^{\mathrm{e}}$ éd. élargie (Vienne : Universal, 1973). 
B (exemple 3b) à cause des caractéristiques suivantes : une économie quant à la longueur de la ligne et au nombre de notes utilisées; une prédominance accordée au mouvement conjoint; une courbe mélodique avec un ambitus restreint de quinte juste sol-ré accompagné d'une finale en mouvement de broderie (sol-fa-sol); une présence constante tout au long de la composition d'une unité donnée ${ }^{26}$.

En ce qui a trait à la construction mélodique, l'exemple 3a met les spécificités de ce premier cantus d'Image en lien avec la mélodie d'introduction de l'unité « $\mathrm{a}^{1}$ ». Leur comparaison fait ressortir les similitudes suivantes :

1. les deux lignes suivent une courbe en arche même si l'une est inversée par rapport à l'autre;

2. les deux lignes sont tracées à partir des mêmes notes structurelles, soit ré et sol.

Par ailleurs, le premier cantus est caractérisé par :

1. l'usage exclusif du mouvement conjoint;

2. l'emploi de la seconde mineure dans le geste de broderie qui termine la ligne mélodique.

L'occurrence de ce premier cantus structure la section $B$ en une petite forme ternaire, $« b^{1}-b^{2}-b^{1-} »$ (voir schéma formel en appendice). Dans l'unité « $b^{1} »$, représentée aux exemples $3 b$ et $3 c$, l'ensemble des collections de notes employées s'associe au motif fondamental «I.F. ».

Entre les unités « $b^{1}$ » et « $b^{1-}$ » s'insère une unité contrastante nommée « $b^{2} »$, dont une portion est illustrée à l'exemple 4 . Ce contraste est amené par le changement du motif d'ostinato. Se trouvant toujours à la partie de piano, l'ostinato présente un cluster réunissant une série de tétracordes puisés dans la collection de mi sur un rythme de deux croches suivies d'une noire. Le motif mélodique déployé sur deux mesures est calqué sur les parties du deuxième violon et de l'alto (les voix extrêmes des accords). La flèche insérée dans l'exemple 4a met en relief la liaison entre les deux unités, car le tracé mélodique que suivent ces accords est extrait du dernier fragment du premier cantus, mais dans un contour inversé.

L'idée de bâtir du nouveau matériel à partir de ce qui existe caractérise la démarche compositionnelle de Tailleferre et crée constamment une liaison entre deux unités malgré l'aspect fragmentaire de la composition. Dans cette même ligne de pensée, la basse do-sol du piano, présente depuis le début de l'œuvre, est retenue. Cette pédale do-sol du piano, qui alterne avec la note de basse $d o$ du violoncelle, reproduit le motif « I-6 » (exemple 4a). Le lecteur observera l'intervalle caractéristique de septième majeure entre les deux notes $d o$ et $d o$. Sur ces notes pédales, la voix supérieure du célesta déroule une ligne mélodique issue de la gamme $f a$ pentatonique dont l'organisation reformule des multiples motifs « I- 3 » et « I-5 ».

26Sur le plan rythmique, l'activité est aussi réduite à son minimum par la présence d'un patron répétitif emprunté au mode trochaïque de la prosodie grecque. 
a) Fin de l'unité $b^{1} \quad$ Unité $b^{2}$ : plan sonore 3

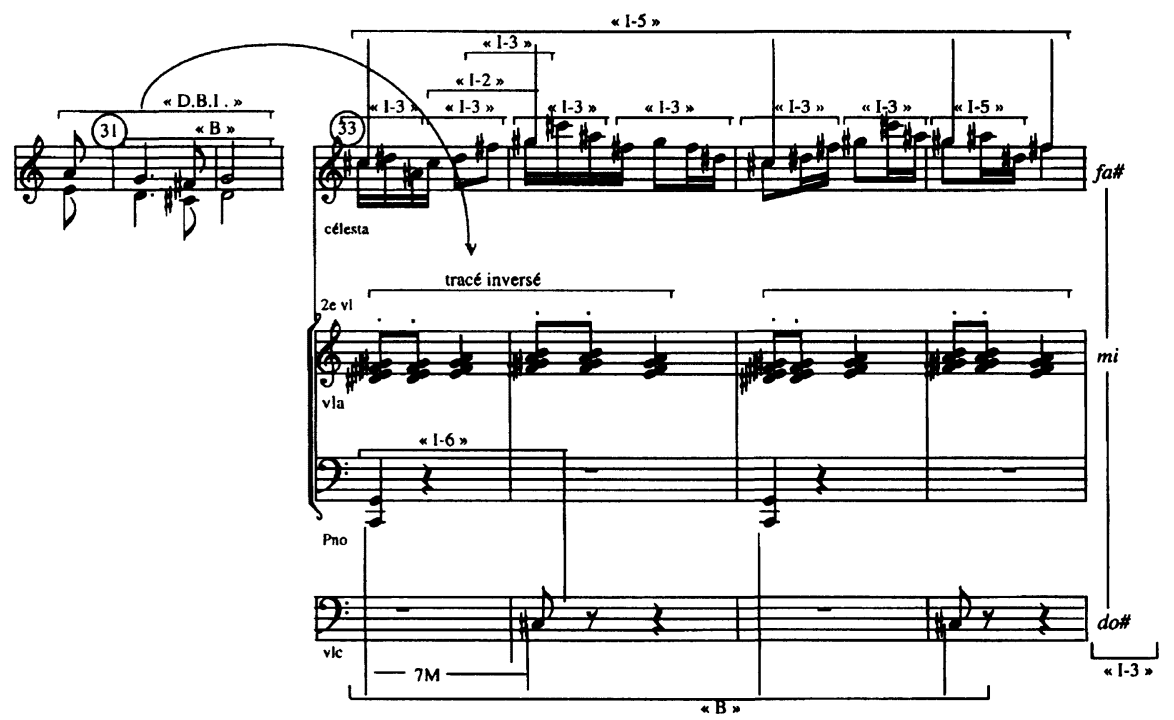

b) Ensembles de notes
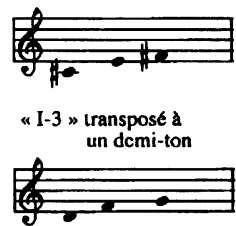

« I-3 » original

Exemple 4 : Motifs caractéristiques de la section $B, 2^{e}$ unité

À l'exception de la double note pédale $d o-s o l$, fixée depuis le début de l'œuvre, les diverses voix de cette unité sont constituées de nouveaux centres : $f a, m i$ et $d o$. Leur union produit l'ensemble « I-3 » à un demi-ton de distance de l'original, comme on le voit à l'exemple $4 \mathrm{~b}$.

Dans l'unité « $b^{1-}$ », qui possède la fonction de réexposition provoquée par le retour du premier cantus, une toute nouvelle mélodie s'annonce au-dessus des éléments linéaires déjà en place. Reproduite à la partie supérieure de l'exemple 5, cette mélodie qui se déploie dans la partie de flûte s'apparente, en tant que structure, à un thème. Ainsi, en attendant la troisième unité de la forme ternaire pour énoncer un thème, Tailleferre rend plus efficace la notion de construction graduelle expliquée antérieurement.

La mélodie thématique de l'unité " $\mathrm{b}^{1-}$ » consiste en deux phrases de huit mesures chacune de construction périodique ${ }^{27}$, ouvrant ainsi un deuxième

27La période classique consiste habituellement en la répétition de deux phrases dont la deuxième, appelée « conséquent », se termine par une cadence plus conclusive (généralement par une cadence 
Unité $b^{\prime \prime}$

a) Mélodie thématique, première partie

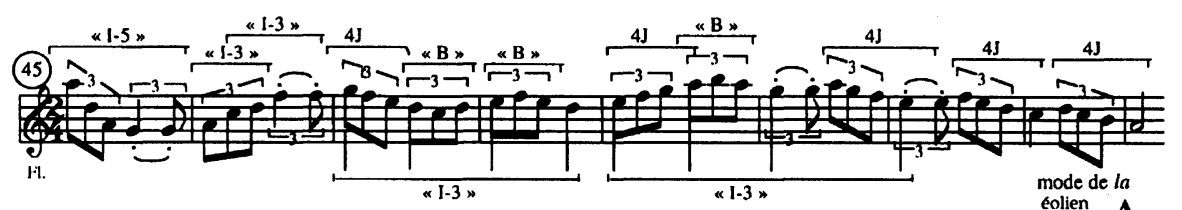

b) Mélodie thématique, deuxième partie

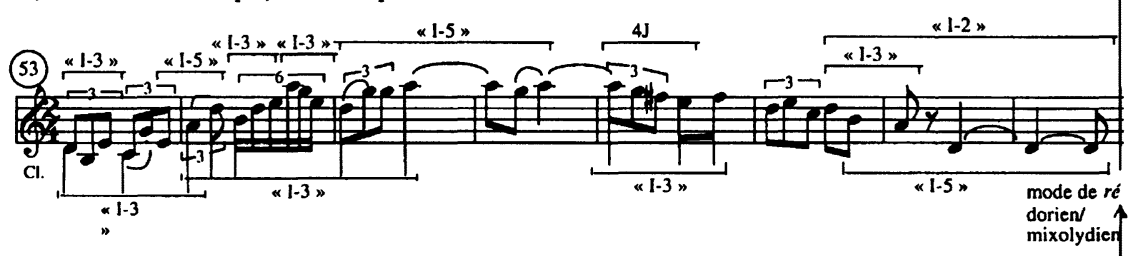

c) $1^{\text {er }}$ cantus

(45)

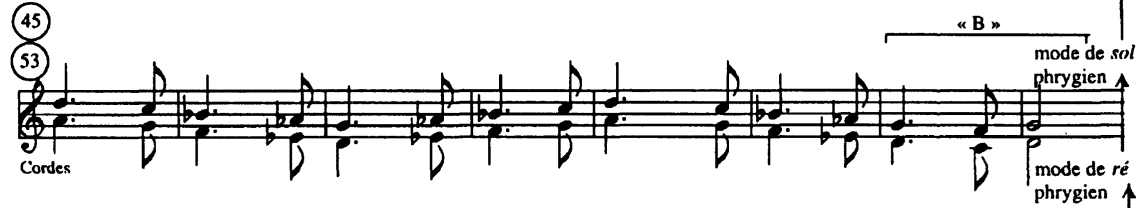

d) Ostinato
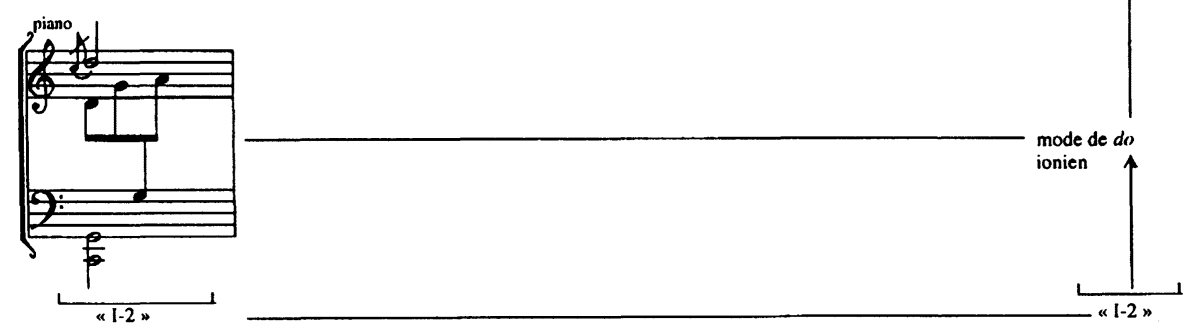

Exemple 5 : Motifs caractéristiques de la section B, $3^{\mathrm{e}}$ unité

dialogue entre la flûte et la clarinette par rapport à l'introduction, mais dans des rôles inversés : la flûte lance, cette fois, le propos thématique (exemple $5 a)$, suivi de la réplique de la clarinette (exemple 5 b). Ces deux lignes mélodiques sont construites à partir de motifs connus, comme le mettent en relief les exemples $5 \mathrm{a}$ et $5 \mathrm{~b}$, et qui surviennent aussi bien à des niveaux de surface que de structure.

authentique parfaite) par rapport à la première, appelée " antécédent », laquelle est ponctuée par une demi-cadence. Pour des explications supplémentaires, voir Schönberg, Fondements de la composition musicale, 43-77. 
L'union des centres de référence des collections de notes utilisées dans les parties mélodiques de l'unité « $\mathrm{b}^{1-}$ » reproduit l'ensemble « I-2 » caractéristique du motif d'ostinato de la partie de piano de la section B (en bas de l'exemple $5)$.

\section{Section $C$}

La section $\mathrm{C}$ débute au plan sonore 6 (schéma formel à l'appendice, mes. 61-76). Elle est soutenue par un motif d'ostinato qui est une variation de celui de la section $B$ puisque l'idée du motif $d$ 'arpège est maintenue dans la voix de basse du piano (exemple 6d).

a) Unité a 1: $1^{\text {re }}$ mélodie

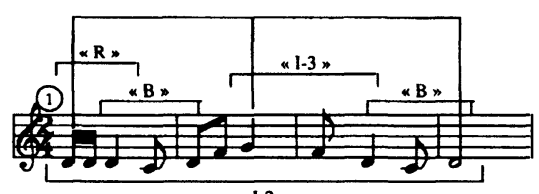

b) Unité b ${ }^{1} 1^{\text {er }}$ cantus

* 1-2*

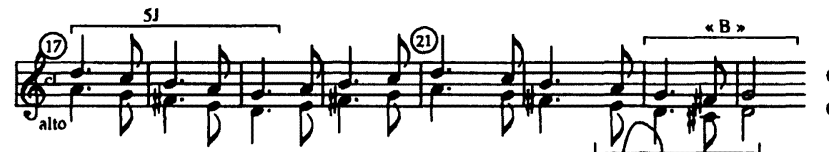

Collection de sol Collection de ré

c) Unité $\mathbf{c}^{1}: 2^{e}$ cantus

d) Ostinato

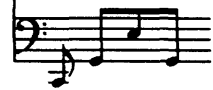

e) Ensembles de notes
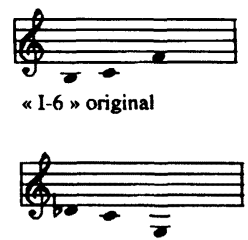

* I-6 \# inversé ct transposé à $2 \mathrm{M}$

Exemple 6 : Motifs caractéristiques de la section C, $1^{\text {re }}$ unité 
La section C représente l'étape finale de la formation d'Image. Un deuxième cantus se trouve à la base de la structure de cette section. La ligne du deuxième cantus s'énonce à la flûte en homorythmie avec la clarinette et les voix supérieures du piano (exemple 6c). Semblable au premier cantus, le matériel du deuxième cantus prend sa source dans la mélodie de l'unité « $\mathrm{a}^{1}$ » (comparer les exemples $6 \mathrm{a}, 6 \mathrm{~b}$ et $6 \mathrm{c})$. De cette mélodie, il conserve le motif « $\mathrm{R}$ » et le motif « B » (comparer les exemples $6 \mathrm{a}$ et $6 \mathrm{c}$ ). Le saut de quinte descendante qui termine la ligne est le renversement de la quarte sol-ré qui structurait la première ligne mélodique.

La ligne de la clarinette dans les huit premières mesures du plan sonore 6 (exemple 6c) suit en général celle de la flûte dans un rapport de quinte juste, et les première et dernière notes de chacune des lignes créent un échange de voix entre les notes ré et sol (exemple 6c). Aussi, l'union des deux voix met en valeur les notes de l'ensemble «I.F. ", do-sol-ré, qui constituent en même temps les points stratégiques de la ligne de la clarinette.

De leur côté, les accords de la partie supérieure du piano proviennent de la collection de ré, soit un demi-ton plus bas que celle de la voix inférieure du premier cantus. Nous verrons plus loin comment le ré occupe une place importante sur le plan de la structure profonde de l'œuvre. La voix supérieure du piano se joint à celles des vents à cause de leur homogénéité rythmique, et non par leur parenté tonale : les trois instruments évoluent dans des collections de notes différentes de $d o$, de sol et de ré. L'union des collections de notes de chacune des parties illustrées à l'exemple $6 \mathrm{c}$ forme l'ensemble « I- 6 » inversé, comme on peut voir à l'exemple 6e.

$\mathrm{Du}$ point de vue formel, le deuxième cantus propre à la section $\mathrm{C}$ sert de point de départ pour une série de trois variations continues. L'exemple 7c montre qu'à chacune des variations, le cantus commence sur une note différente. En les reliant, nous obtenons l'ensemble «I.F. " constitué des notes do-ré-sol caractéristiques du deuxième cantus (exemple $7 \mathrm{~b}$ ).

Une mélodie thématique s'ajoute lors de la première variation du cantus. L'exemple $7 \mathrm{a}$ indique que le thème présenté à la première variation ne reviendra que lors de la troisième variation, ce qui amène à regrouper par paires les premières unités de la section $\mathrm{C}$. Les éléments de chacune des paires sont désignés par $« \mathrm{c}^{1}$ »et $« \mathrm{c}^{1-} »$ respectivement.

Sous les divisions formelles de l'exemple 7 a se trouvent mentionnés les types d'ensembles formés par la combinaison des différentes collections propres à chacune des unités. La présence constante de l'ensemble «I-6» tout au long des deux unités $" \mathrm{c}^{1}$ » et $" \mathrm{c}^{1-}$ » ne peut nous échapper, et ce, malgré la diversité des collections dont les noms se trouvent entre parenthèses. Deux d'entre eux utilisent les mêmes collections ré-do-sol et correspondent respectivement à la première partie des deux unités $\left\langle\mathrm{c}^{1}\right.$ » et $\left\langle\mathrm{c}^{1-}\right.$ ».

La dernière étape d'élaboration d'Image se manifeste réellement dans la dernière unité de la section $\mathrm{C}$. L'unité $~ « \mathrm{c}^{2}$ » rassemble tous les instruments qui se partagent les fonctions mélodique et d'accompagnement. Les parties mélodiques débutant à la mesure 93 de l'exemple 8a comportent un contrepoint 
a) Division formelle de la section C

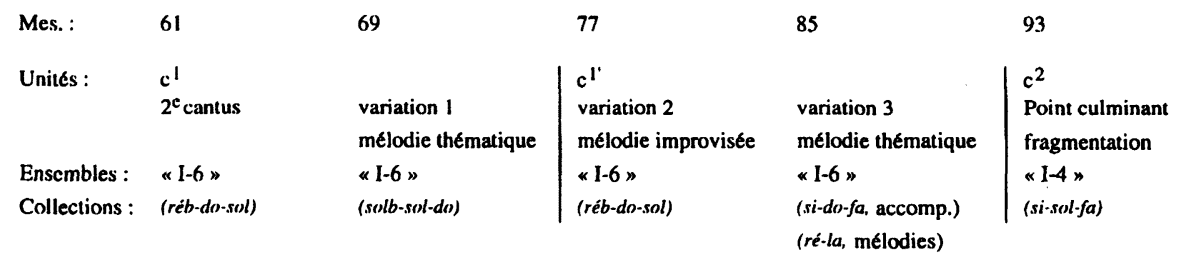

b) Unité $\mathrm{c}^{1}$

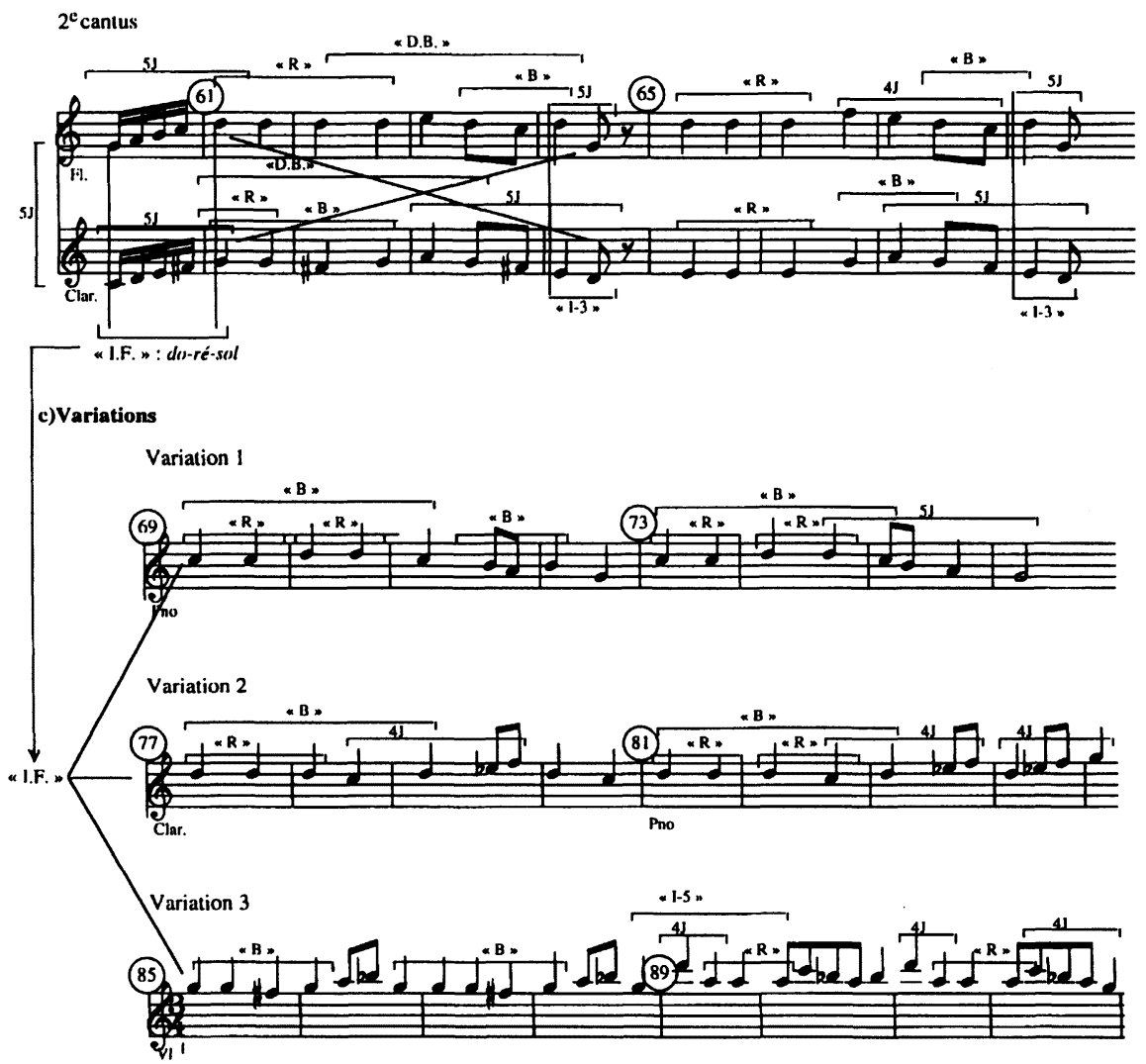

Exemple 7 : Forme et motifs caractéristiques de la section $\mathrm{C}$

renversable entre la flûte et la clarinette. Le matériel mélodique utilisé fait référence à une unité antérieure, précisément à l'unité « $b^{2}$ ».

Pour sa part, le matériel d'accompagnement retranscrit à l'exemple $8 \mathrm{~b}$ est réduit au seul motif « $\mathrm{B}$ » harmonisé à plusieurs voix. Les collections de notes utilisées dans chacune des parties d'harmonisation reproduisent l'ensemble « I-4 » et ont respectivement comme centre de référence les notes $f a$, sol et si. 


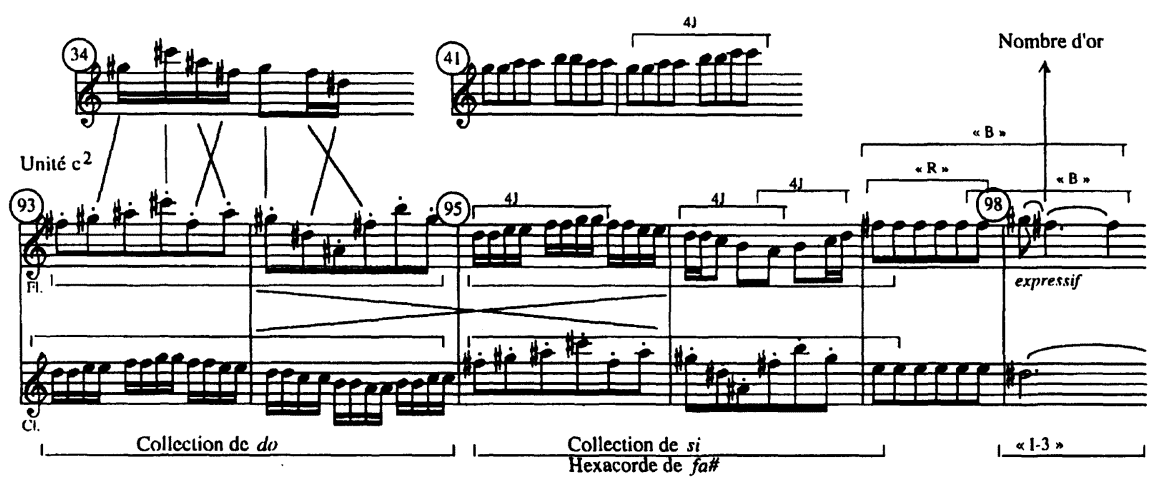

b) Parties d'accompagnement
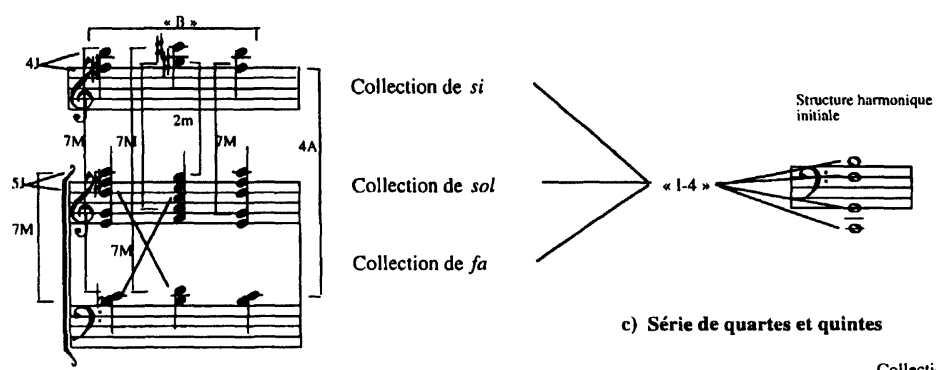

c) Série de quartes et quintes

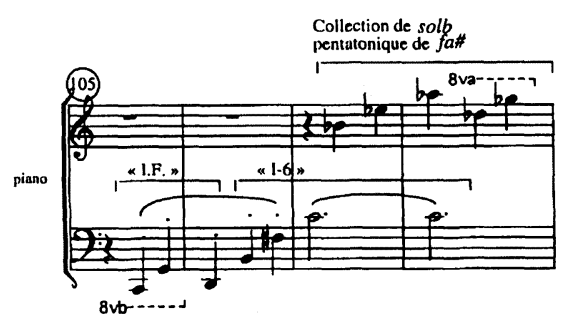

Exemple 8 : Motifs caractéristiques de la section $\mathrm{C}$, dernière unité

Ces dernières correspondent précisément aux trois des quatre notes incluses dans la structure harmonique initiale.

La section $C$ est réellement pensée en fonction de l'atteinte du point culminant du processus d'élaboration. Le tutti, le chaos sonore provoqué par les clusters ainsi que les multiples frictions harmoniques amenées par la polytonalité contribuent tous à souligner davantage ce moment fort de l'œuvre. Cette tension se construit jusqu'à l'indication expressif de la mesure 98 (exemple 8a), une mesure d'autant plus significative qu'elle équivaut au «nombre d'or ${ }^{28}$ de l'ensemble des 159 mesures de l'œuvre. 
Une appoggiature constituée des notes sol et $f a$ caractérise cette portion de la section. Ce geste sol-fa possède un lien avec la broderie de la mélodie initiale, constituée à l'origine des notes ré et do (exemple 1a). Il n'est pas étonnant que ces deux dyades soient à une distance de quarte augmentée, intervalle caractéristique de l'œuvre. De plus, ce même lien de quarte augmentée est repris à la mesure 98 entre le geste d'appoggiature sol-fa et la broderie $r e ́-d o$ qui, d'après l'exemple $8 \mathrm{~b}$, se trouve, entre autres, dans la voix supérieure de la clé de $f a$.

De la mesure 98 jusqu'à la fin du premier stade, la compositrice ne se restreint qu'à l'essentiel du matériel motivique-harmonique antérieur. Les dernières mesures ne serviront qu'à ponctuer une série de quintes et de quartes mélodiques. L'exemple 8c montre que cette série, projetée par le son du piano, débute notamment avec l'ensemble «I.F. » do-sol-ré, suivi de l'ensemble « I-6 » et d'une série de quartes produisant la collection pentatonique de sol, soit l'enharmonique de la collection de $f a$ utilisée antérieurement.

\section{Structure sous-jacente de l'œuvre}

À partir du deuxième stade de la composition, le compte à rebours est enclenché, ce deuxième stade étant, comme nous l'avons déjà mentionné, l' « image » du premier stade, soit sa reproduction inversée. Aussi, le tissu sonore opaque qui s'était graduellement formé va maintenant se désagréger au fur et à mesure que nous arriverons à la fin de l'œuvre.

Tailleferre débute le deuxième stade par une fausse réexposition en présentant le premier cantus de la section $\mathrm{B}$ à partir de la note do qui se déploie sur une double pédale ré-la. L'harmonie ré-la-do est représentée à la mesure 110 dans le graphique $B$ se trouvant en appendice. Cette fausse réexposition permet au niveau le plus profond de l'œuvre d'exercer un geste de broderie à un demi-ton de distance de la note $d o$ qui soutient chacune des sections extrêmes de la composition.

Ce geste de broderie do-ré-do est la reproduction élargie, à l'échelle de l'œuvre (graphique B, mes. 1, 110 et 138), du geste de broderie de la voix de basse qui articule la structure de la forme ternaire de la section B, mais en utilisant cette fois les notes do-do-do (graphique B, mes. 13, 33 et 45). $\mathrm{Au}$-dessus de cette voix de basse, la voix supérieure effectue un contrepoint en mouvement contraire résultant en un geste de broderie sur les notes ré-do-ré. Ce geste de broderie structurel avait été anticipé dans la section A en reliant chacune des premières notes mélodiques des unités « $\mathrm{a}^{1}$ » et « $\mathrm{a}^{2}$ » (graphique $B$, mes. 1 et 9). La broderie incomplète ainsi représentée est reprise à l'intérieur de la section $C$ aux mesures 61 et 103 de la voix supérieure du graphique $B$.

Ce geste ré-do couvrant tout le premier stade (mes. 1-109) contraste avec le geste ré-do-ré (graphique B, mes. 1, 110,138 et 159) à l'échelle de l'œuvre. Les deux modes majeur et mineur de la seconde appliqués à la broderie s'opposaient déjà l'un à l'autre selon les finales apposées à la ligne du premier cantus. L'exemple 3 témoigne des deux modes de finales utilisés dans les deux lignes mélodiques. 
À ce geste de broderie mélodique au niveau profond de l'œuvre (ré-do-ré, mes. $1,110,138$ et 159 ) se joint une troisième voix créée par la note sol qui se manifeste tout d'abord au cœur de la section B (mes. 25). Ce sol, qui s'associe au premier cantus, forme avec les deux autres notes structurelles de la section $\mathrm{B}$, ré et $d o$, l'ensemble « I- 6 » que nous avons clairement mis en évidence tout au long de cette démonstration.

Ce même motif est reproduit à un demi-ton inférieur au moment de l'enchâ̂nement des deux stades de la composition avec les notes $d o-f a-d o$ (mes. 103, 108 et 110). Ce lien du demi-ton est caractéristique de la signature compositionnelle de Tailleferre à ce point précis de réexposition de la composition.

Dans le deuxième stade, cette troisième voix se démarque encore davantage avec la note sol. Cette dernière forme, avec les notes $d o-r e ́, l$ 'ensemble «I.F. », qui représente la structure mélodique fondamentale dont les notes figurent en blanches (mes. 110 et 159). De plus, cette structure mélodique fondamentale est la reproduction, au niveau profond, de la ligne structurelle propre à la toute première mélodie de l'œuvre, comme nous l'avons vu à l'exemple 1 .

Depuis le début de l'œuvre, Tailleferre n'a cessé d'afficher, dans chacune de ses sections formelles, la présence des trois notes, ré-do-sol, de la structure mélodique fondamentale que nous avons nommée « idée fondamentale ». À l'intérieur de cette ligne fondamentale, le geste de broderie se démarque tout particulièrement. Sa présence s'est faite constante tout au long de l'œuvre. De plus, comme l'indique l'exemple $1 \mathrm{~b}$, l'œuvre se termine en reproduisant à la dernière mesure, dans une activité rythmique très rapide et dans une étendue de voix de deux octaves, les notes du mouvement de broderie initial : le geste do-ré-do projeté à la mesure 159 est l'« image » du geste ré-do-ré de la mesure 1, c'est-à-dire sa représentation inversée.

\section{Conclusion}

Voilà où se trouve la valeur sémantique et cohésive de l'œuvre. Le court motif de broderie ré-do-ré, ouvrant la première mélodie de la section A d'introduction, sert de grand diviseur en deux parties dans une proportion qui se rapproche du 2:1 (appendice, partie supérieure, et graphique B, mes. 1, 110 et 159). À l'intérieur de cette division, le matériel thématique-mélodique est structuré en forme d'arche tripartite dans une proportion quasi égale en termes de nombre de temps (appendice, partie supérieure). Les deux types de cantus, représentant les sections $B$ et $C$ respectivement, sont responsables de cette coupe formelle tripartite ayant en plus comme cadre la section $\mathrm{A}$.

Sous le grand mouvement de broderie ré-do-ré de la voix mélodique résultant de la coupe tripartite (graphique B, mes. 1, 110 et 159), la ligne de la basse effectue un autre mouvement de broderie avec les notes do-ré-do; ce dernier est presque la représentation inversée, à l'exception du bémol appliqué à la note ré. En plus, la rencontre des deux notes de broderie ré et do à la mesure 110 crée l'intervalle caractéristique de septième majeure présente dans le premier accord de l'œuvre (exemple 1). Aussi, nous pouvons maintenant comprendre comment l'« idée fixe » dont parle Tailleferre suit constamment 
le développement de l'œuvre, de la première mesure jusqu'à la dernière, et ce, à plusieurs niveaux de la composition.

Cette démonstration, faite à partir de la méthode d'analyse motivique tant au niveau profond que de surface, nous a permis non seulement d'offrir un modèle pour étudier les ouvres diatoniques et non tonales du début du $\mathrm{XX}^{\mathrm{e}}$ siècle, mais aussi et surtout de constater la cohésion compositionnelle dont Tailleferre arrive à faire preuve dans une musique qui dégage en premier lieu une impression de spontanéité. 


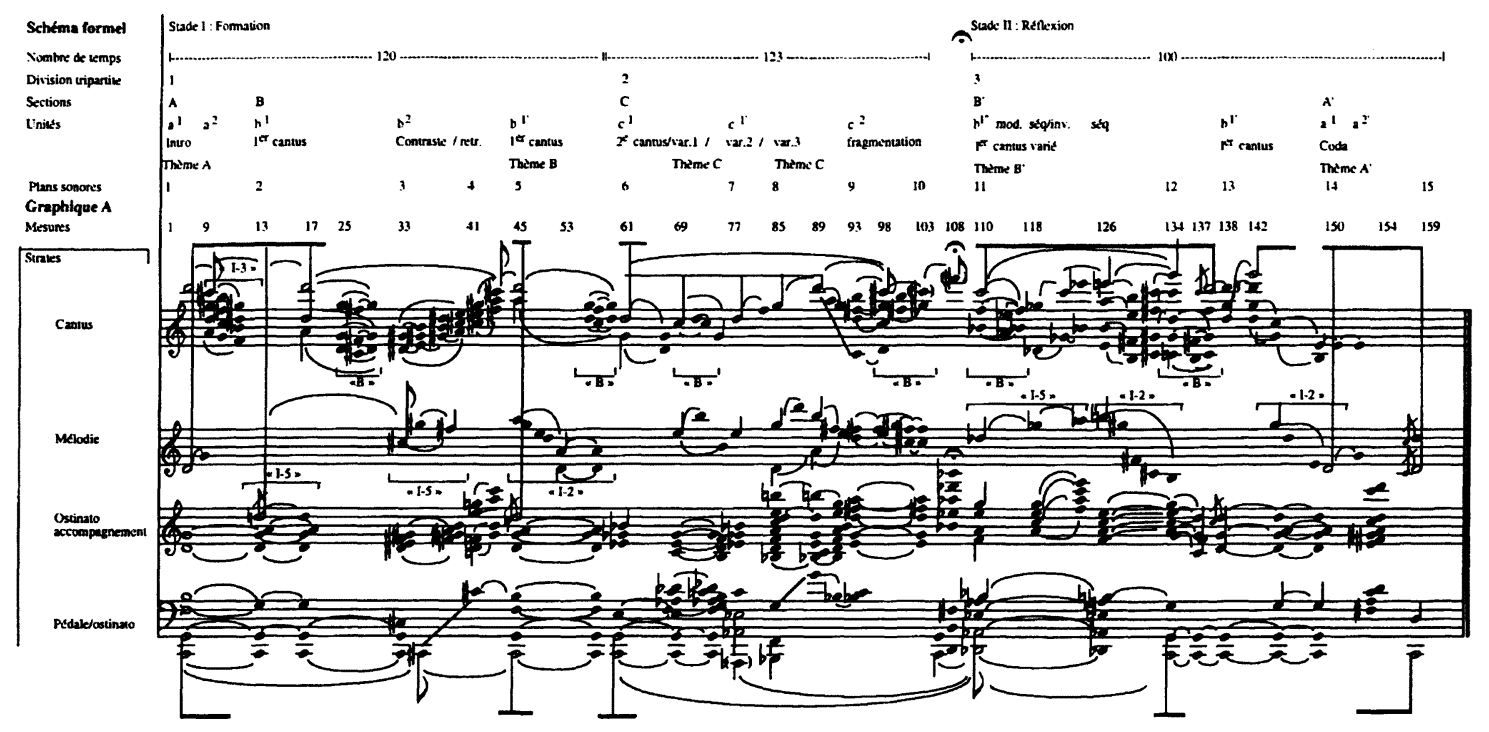

Graphique B

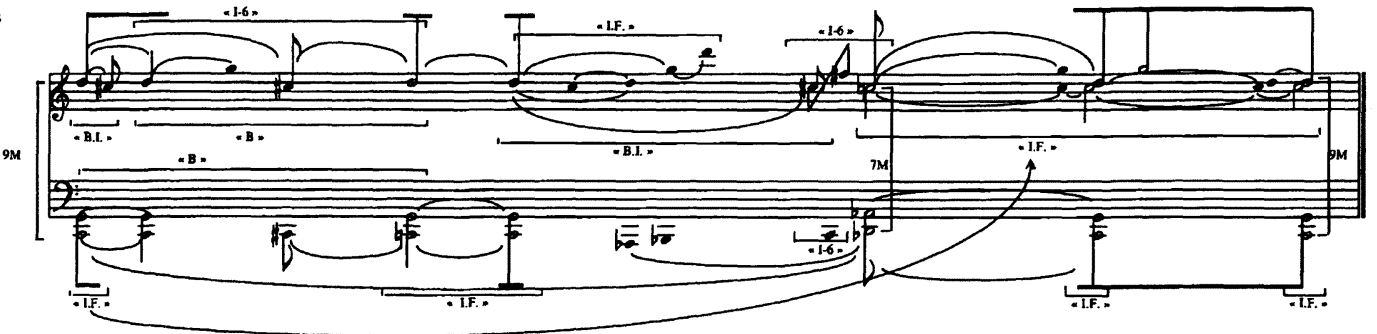




\section{Résumé}

L'article présente une étude analytique d'Image pour huit instruments (1918) de Germaine Tailleferre. La démonstration fera la preuve de la cohésion compositionnelle du travail de Tailleferre en se penchant sur deux aspects fondamentaux : le motif et la forme. La démarche analytique proposée consiste à considérer le motif sur divers plans de la composition. La représentation graphique des structures linéaires qui résulte de l'analyse permet d'illustrer comment les notes du motif initial de surface se tiennent à l' arrière-plan de la composition, lesquelles notes s'enchaînent en synchronisme avec les diverses composantes de la structure formelle de l'œuvre. 